\title{
The Pigtail Sign: A New Spectral Domain Optical Coherence Tomography Finding After Inverted ILM Flap Surgery for Idiopathic Macular Hole
}

This article was published in the following Dove Press journal: Clinical Ophthalmology

\author{
Samir Mohamed El Baha \\ Omar Elbaha \\ Mahmoud A Abouhussein \\ Ophthalmology, Alexandria University, \\ Alexandria, Egypt
}

Purpose: To report a new spectral domain optical coherence tomography (OCT) finding called the pigtail sign. It was found during follow-up of idiopathic macular hole cases after inverted internal limiting membrane (ILM) flap surgery.

Methods: This is a retrospective case series of 12 eyes (12 patients) who underwent inverted ILM flap technique for idiopathic macular hole closure. Spectral domain optical coherence tomography images were analyzed for the presence of this new finding.

Results: A new spectral domain OCT finding of a curved coiled line hanging above the surface of the retinal layers was seen in all patients. The mean preoperative best corrected visual acuity (BCVA) $\log$ MAR value was $1.25 \pm 0.11$. At the 6 -month follow-up visit, the mean LogMAR BCVA was $0.35 \pm 0.18$. All the cases showed macular hole closure after surgery.

Conclusion: This new spectral domain OCT sign can be seen in some OCT scans during follow-up of idiopathic macular hole cases after inverted ILM flap surgery.

Keywords: inverted ILM flap, macular hole, pigtail sign

\section{Introduction}

Idiopathic macular hole is one of the main causes of loss of central vision due to vitreomacular interface pathology. It consists of a foveal defect associated with sensory neural retinal interruption exposing the retinal pigment epithelium. ${ }^{1}$ The general prevalence of idiopathic macular hole can vary from 0.2 to 3.3 per $1000 .^{2,3}$

Vitrectomy with internal limiting membrane (ILM) peeling is the main surgical procedure for idiopathic macular holes. It relieves tangential traction surrounding the macular hole leading to hole closure with less incidence of postoperative epiretinal membrane formation. ${ }^{4}$ In challenging cases, like high myopic macular holes and idiopathic macular holes exceeding $400 \mu \mathrm{m}$, the rate of macular hole closure is much less after vitrectomy with ILM peeling. ${ }^{5}$ Moreover, these cases may show postoperatively a flat open macular hole with bare retinal pigment epithelium and low best corrected visual acuity. ${ }^{6}$

To overcome these problems, the inverted ILM flap technique was introduced for treating large idiopathic macular holes and high myopic holes. This technique was shown to increase the rate of large idiopathic holes closure. It also showed improved foveal anatomy as shown by optical coherence tomography and the flat open appearance did not occur. ${ }^{7}$
Correspondence: Mahmoud A Abouhussein Ophthalmology, Alexandria University, 8 Hassan Allam Street, Kasr Elmoltazem Building, Smouha II03, Alexandria, Egypt Tel +20 I00I026075

Email nad03alaa@yahoo.com 
The exact mechanism of macular hole closure after inverted ILM flap technique is not completely understood. The proposed theory is that Muller cells in the ILM induce proliferation of glial tissue while acting as a scaffold for proliferation of neuroretinal tissue filling the hole leading to closure. ${ }^{8}$ ILM is also a basement membrane needed for glial cellular proliferation to fill a large macular hole. ${ }^{9}$

The original technique of inverted ILM flap involved tucking the inverted flap inside the macular hole. ${ }^{7}$ The surgical manipulations for this step can potentially cause retinal pigment epithelium damage affecting the final visual outcome. It was reported that just covering the macular hole with the inverted ILM flap without extra manipulations was enough to achieve a successful closure. ${ }^{10}$

Different studies have described the optical coherence tomography (OCT) changes that occur after surgery of idiopathic macular hole cases. The main factors that affected the final functional outcome were the type of macular hole closure, foveal contour, ellipsoid zone defects, external limiting membrane discontinuity, and affection of nerve fiber layer postoperatively. ${ }^{6,11-13}$

A recent comparative study showed that surgical manipulations to tuck the ILM in the hole and the ILM massage are not necessary during the inverted ILM technique. The group without extra flap manipulations achieved good anatomic and visual outcomes. The authors concluded that the modified technique without extra flap manipulations seems to be safe and easy to perform, and it could reduce the risks of iatrogenic damage with large idiopathic macular holes. ${ }^{14}$

We describe a group of patients with idiopathic macular holes who underwent vitrectomy with inverted ILM flap technique without extra manipulations. During followup, patients were imaged with spectral domain OCT. A subgroup of our patients showed a new OCT finding, which we termed the "pigtail" sign: Part of the ILM was detected in some OCT scans as a curved line protruding from the retinal surface into the vitreous cavity with tapered and coiled distal end, similar to a pig's tail.

\section{Patients and Methods}

This is a retrospective study based on the review of medical records of 200 patients with idiopathic large macular holes (more than 400 microns in diameter) after inverted ILM flap surgery. From these, we included in the study 12 patients that showed the new OCT sign during follow-up.

Alexandria University ethics review board approval was obtained and the research followed the tenets of the
Declaration of Helsinki. All patients included in this study provided informed consent prior to surgery. As this study consists of patient unidentified data, no consent was required for data collection or reporting.

Patients underwent vitrectomy with inverted ILM flap technique without extra manipulation. All cases were operated upon by a single surgeon (S.M.E.) using the same technique. Minimum duration of follow-up was 6 months.

Exclusion criteria were secondary macular holes, idiopathic macular holes less than 400 microns, conditions affecting the retina such as diabetic retinopathy, high myopia, age-related macular degeneration, and history of intraocular operations other than cataract surgery.

Complete preoperative evaluation was done, including duration of symptoms, best corrected visual acuity (BCVA) measurement, fundus biomicroscopy using 78-D non-contact lens. The best-corrected visual acuity was converted to a logarithm of minimal angle of resolution for statistical analysis. OCT was done using commercially available spectral domain OCT: Cirrus HD-OCT (Carl Zeiss Meditec, Dublin, CA, USA).

\section{Surgical Technique}

All cases were operated upon by the Constellation vision system (Alcon-USA) 23-gauge system. All phakic patients underwent simultaneous phacoemulsification at the time of vitrectomy. Complete 23 gauge transconjunctival vitrectomy with removal of posterior hyaloid face was done. The ILM was stained by injection of $0.5 \mathrm{~mL}$ of Brilliant Blue G Solution 0.025\% (ILM-BLUE- DORC, The Netherlands) gently into the vitreous cavity. The stain was left in contact with the retinal surface for 1 minute to achieve adequate staining. The ILM was then peeled in all patients in a circular fashion. ILM was left attached to the macular hole edges without any extra manipulations. Fluid air exchange was then performed taking care that air exerts pressure one side of the ILM funnel to cover the hole. Air was then replaced with $20 \%$ mixture of sulfur hexafluoride (SF6). Patients were asked to keep a facedown position postoperatively for 5 days.

During follow-up visits, patients were subjected to complete ocular examination and spectral domain OCT evaluation (Cirrus HD-OCT Carl Zeiss Meditec). Evaluation of OCT scans was done with stress on the ellipsoid zone, external limiting membrane, retinal pigment epithelium, and the presence or absence of the pigtail sign. 
Charts of patients showing the pigtail sign were retrospectively reviewed. Different parameters were recorded including age, sex, macular hole diameter, BCVA at different time points, and time of detection of the pigtail sign.

Statistical analysis was done using Statistical Package for Social Sciences (SPSS, Version 20, IBM Corporation, Armonk, NY, USA) software. Paired $t$-test was used for comparisons between different means. Probability value less than 0.05 was considered to be significant.

\section{Results}

A retrospective chart review of 200 patients who underwent inverted ILM flap technique without extra manipulations as a surgical treatment for large idiopathic macular holes was done. From these, 12 patients that showed the pigtail sign during follow-up OCT scans were included in the study.

Table 1 summarizes the baseline characteristics of the included patients. There were eight females and four males. The mean age of the patients was $62.83 \pm 2.79$ years (range, 60-67 years). Eight patients were phakic, and four patients were pseudophakic. The mean duration of symptoms before presentation was $6.16 \pm 3.18$ weeks (ranging from 2 to 12 weeks). The mean preoperative BCVA (logMAR value) was $1.25 \pm 0.11$ (ranging from 1 to 1.3 ). The Snellen visual acuity ranged from $20 / 400$ to 20/200. Preoperative OCT showed that the mean minimal macular hole diameter was $623.16 \pm 152.32 \mu \mathrm{m}$ (ranging from 407 to $846 \mu \mathrm{m}$ ). The mean duration of follow-up was $9.83 \pm 3.02$ months (ranging from 6 to 15 months).

Table I Baseline Characteristics of the 12 Included Patients

\begin{tabular}{|l|l|}
\hline $\begin{array}{l}\text { Gender, no. (\%) } \\
\text { Males }\end{array}$ & $4(33.3 \%)$ \\
Females & $8(66.6 \%)$ \\
\hline Age (years) (mean \pm SD; range) & $62.83 \pm 2.79(60-67)$ \\
\hline $\begin{array}{l}\text { Eye, no. (\%) } \\
\text { Right eye }\end{array}$ & $4(33.3 \%)$ \\
Left eye & $8(66.6 \%)$ \\
Preoperative BCVA (logMAR) (mean \pm & $1.25 \pm 0.11$ (I- I.3) \\
SD; range) & $623.16 \pm 152.32$ \\
Macular hole diameter ( $\mu$ m) (mean \pm SD; & $(407-846)$ \\
range) & $6.16 \pm 3.18(2-12)$ \\
Duration of symptoms (weeks) (mean \pm & \\
SD; range) & \\
\hline Baseline lens status, no. (\%) & $8(66.6 \%)$ \\
Phakic & $4(33.3 \%)$ \\
Pseudophakic & $9.83 \pm 3.02(6-15)$ \\
Follow-up (months) (mean \pm SD; range)
\end{tabular}

The operative data of all 12 patients showed that surgery was done successfully with no recorded intraoperative complications. All 12 patients were operated upon once for the macular hole.

During follow-up of these cases, some OCT scans showed remnants of the ILM as a curved line protruding from the retinal surface into the vitreous cavity with tapered and coiled distal end. We chose the term pigtail sign to describe this OCT appearance. The pigtail sign was detected on the nasal side of the scans in seven patients, over the center of the fovea in two patients, and on the temporal side in three patients. The mean time of the first detection of this pigtail appearance was $4.66 \pm 1.88$ weeks postoperatively (ranging from 2 to 8 weeks). Figures 1-4 show examples of OCT scans of the pigtail sign. Clinical fundus examination did not show any clue to the presence of pigtail appearance of the ILM in any of the cases. Reaching the 6th month follow-up visit, this OCT sign was not detected in any of the 12 patients. The mean

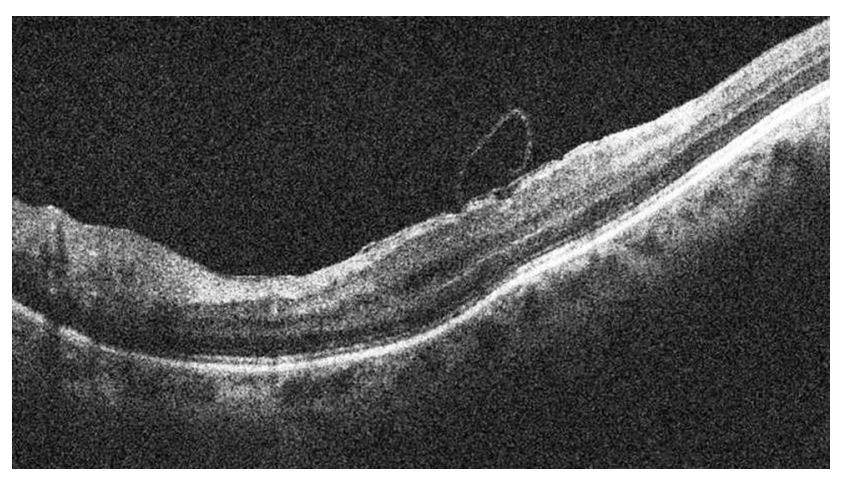

Figure I OCT scan of the left eye of a patient showing the pigtail sign on the nasal side of the scan at the 2-week visit.

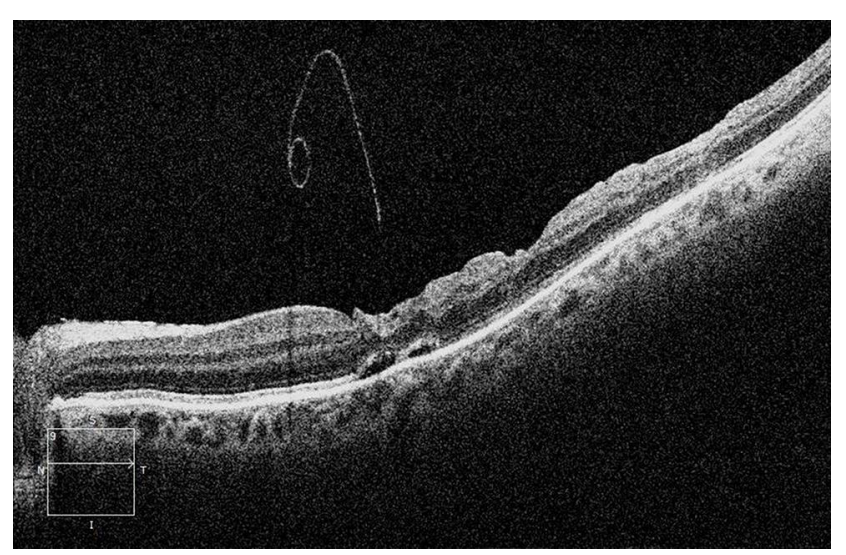

Figure 2 OCT scan of the left eye of a patient showing the pigtail sign on the nasal side of the scan at the 4 -week visit. 


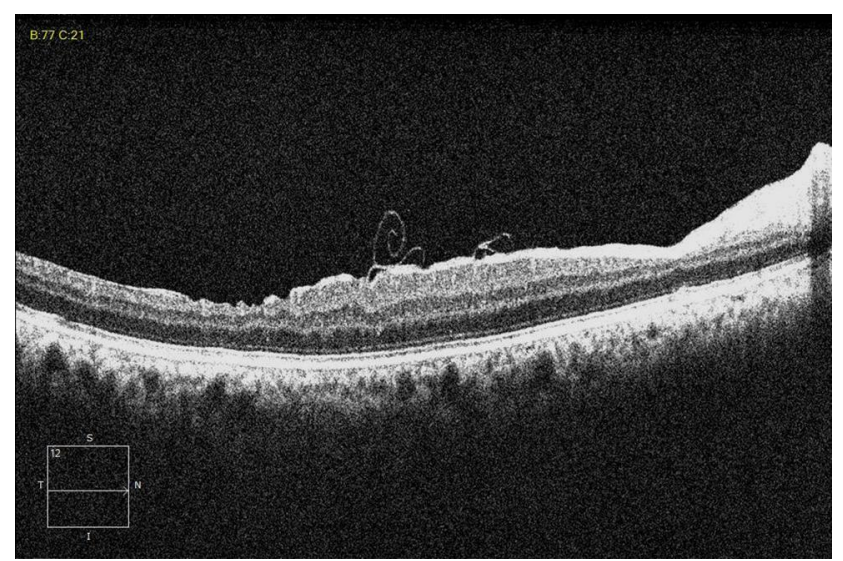

Figure 3 OCT scan of the right eye of a patient showing the pigtail sign on the temporal side of the scan at the 6-week visit.

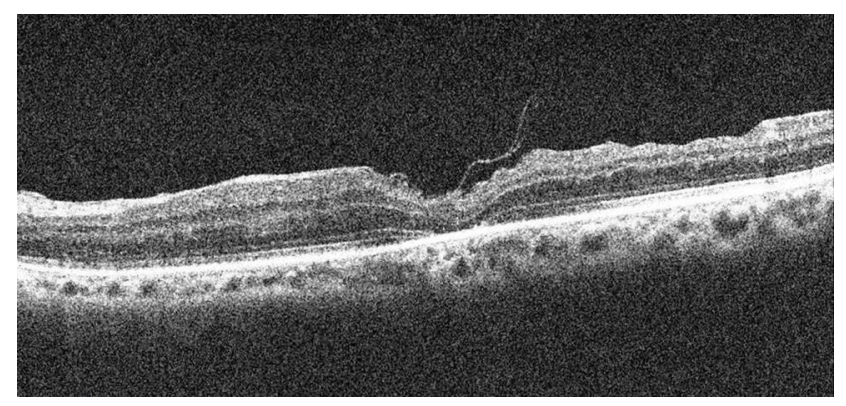

Figure 4 OCT scan of the left eye of a patient showing the pigtail sign over the fovea at the 8-eek visit.

duration of pigtail disappearance is 18.66 weeks (range 16-24 weeks).

All patients showed macular hole closure at the 6-month follow-up OCT scans. The U-type closure (normal foveal contour) was recorded in nine eyes and the V-type closure (steep foveal contour) was recorded in three eyes. External limiting membrane defects were detected in two patients, while ellipsoid zone defects were noticed in four patients.

At the 6-month follow-up visit, the mean LogMAR best corrected visual acuity was $0.35 \pm 0.18$ (ranging from 0.1 to 0.7 ). The Snellen visual acuity ranged from $20 / 100$ to $6 / 7.5$. These results were statistically significant compared to preoperative values, with a $P$-value of $\leq$ 0.001. All 12 patients showed improvement in their best corrected vision.

\section{Discussion}

Inverted ILM surgery for idiopathic macular holes is a very popular surgery nowadays. Since the original technique was described, ${ }^{7}$ several modifications have been reported. One of these modifications was to limit the area of ILM peeling to the temporal side only. ${ }^{13}$ Another reported technique was to use perfluoro-n-octane to fix the ILM flap in place. ${ }^{15}$ There was also another study using autologous transplantation of the ILM with help of a low-molecular-weight viscoelastic agent. ${ }^{16}$

All of these techniques included steps of flap manipulations and tucking inside the macular hole with the possibility of iatrogenic damage of the ILM flap or injury to the retinal pigment epithelium at the base of the macular hole. ${ }^{10}$ One study showed the enlargement of retinal pigment epithelium atrophy following the inverted ILM flap surgery in a large longstanding idiopathic macular hole. ${ }^{17}$

Recently, it was shown that only covering of the macular hole with the inverted flap is needed. Extra flap manipulations were not needed to achieve anatomic closure of the hole with good functional outcome. ${ }^{14}$

We retrospectively reviewed our 200 cases of inverted ILM surgery for large idiopathic macular holes. In our group of patients, the inverted ILM flap technique was done without further manipulations of the flap.

This study aimed to describe a specific OCT sign that resulted from ILM remnants after inverted ILM flap surgery without manipulations. Part of the ILM was detected in some OCT scans as a curved line protruding from the retinal surface into the vitreous cavity with tapered and coiled distal end, similar to a pig's tail. This OCT finding was detected in the early follow-up period. It was recorded as early as 2 weeks postoperatively. This is a temporary appearance that disappeared in the following period and was not detected by 6 months postoperatively.

Remnants of the ILM on the surface of the retina were pointed to in previous studies. Following the original inverted ILM flap technique that involved tucking the flap inside the macular hole, Boninska et $\mathrm{al}^{18}$ observed hyperreflective remnants of the ILM on the retinal surface in 66 cases (44.29\%). It was visible early in the follow-up. This hyperreflective tissue over the macula had fixed appearance over time and did not affect the final visual outcome. ${ }^{18}$ This is to be expected following tucking of the folded flap in the macular hole resulting in a multilayered membrane.

Following the inverted ILM flap without manipulations, Casini et $\mathrm{al}^{14}$ noticed remnants of the ILM flap near the fovea in some of their patients. These patients achieved similar visual outcome to the rest of the cohort. ${ }^{14}$ This phenomenon was noticed in the early postoperative period and disappeared by 6 months. This is the same time 
frame noticed in our study because we were using the same surgical technique.

As we would expect, no reports of similar findings were recorded in studies after complete ILM peeling for idiopathic macular holes.

The remnants of the ILM can appear in different shapes on OCT scans. One of these shapes, which we are describing in our study, is the pigtail appearance: The ILM remnants appear as a curved line protruding from the retinal surface into the vitreous cavity with tapered and coiled distal end. All of the included patients achieved anatomic closure of the hole with statistically significant improvement in visual acuity. Our surgical technique does not involve tucking of the ILM flap inside the hole. This probably allowed for large parts of the ILM to be hanging on the surface of the retina causing this OCT appearance.

In conclusion, this study described a specific OCT sign that was found in the follow up of idiopathic macular hole patients after inverted ILM flap technique without extra manipulations. This is the first study, to the best of our knowledge, to describe this pigtail sign in detail. This new OCT sign gives new perspective in the follow-up of idiopathic macular hole cases after inverted ILM flap surgery.

\section{Disclosure}

Mahmoud Abouhussein reports a patent: The pigtail sign, pending. The authors report no grants or funds, no proprietary interest, and no other conflicts of interest for this work.

\section{References}

1. Duker JS, Kaiser PK, Binder S, et al. The international vitreomacular traction study group classification of vitreomacular adhesion, traction, and macular hole. Ophthalmology. 2013;120(12):2611-2619. doi:10. 1016/j.ophtha.2013.07.042

2. Casuso LA, Scott IU, Flynn HW Jr, et al. Long-term follow-up of unoperated macular holes. Ophthalmology. 2001;108(6):1150-1155. doi:10.1016/s0161-6420(01)00581-4

3. Ezra E. Idiopathic full thickness macular hole: natural history and pathogenesis. Br J Ophthalmol. 2001;85(1):102-108. doi:10.1136/ bjo.85.1.102
4. Vaziri K, Schwartz SG, Kishor KS, et al. Rates of reoperation and retinal detachment after macular hole surgery. Ophthalmology. 2016;123(1):26. doi:10.1016/j.ophtha.2015.09.015

5. Kuriyama S, Hayashi H, Jingami Y, Kuramoto N, Akita J, Matsumoto M. Efficacy of inverted internal limiting membrane flap technique for the treatment of macular hole in high myopia. Am J Ophthalmol. 2013;156(1):125-131.e1. doi:10.1016/j.ajo.2013.02.014

6. Imai M, Iijima H, Gotoh T, Tsukahara S. Optical coherence tomography of successfully repaired idiopathic macular holes. Am J Ophthalmol. 1999;128(5):621-627. doi:10.1016/S0002-9394(99) 00200-7

7. Michalewska Z, Michalewski J, Adelman RA, Nawrocki J. Inverted internal limiting membrane flap technique for large macular holes. Ophthalmology. 2010;117:2018-2025.

8. Spiteri Cornish K, Lois N, Scott N, et al. Vitrectomy with internal limiting membrane (ILM) peeling versus vitrectomy with no peeling for idiopathic full thickness macular hole (FTMH). Cochrane Database Syst Rev. 2013;5(6):Cd009306.

9. Oh J, Yang SM, Choi YM, Kim SW, Huh K. Glial proliferation after vitrectomy for a macular hole: a spectral domain optical coherence tomography study. Graefes Arch Clin Exp Ophthalmol. 2013;251 (2):477-484. doi:10.1007/s00417-012-2058-9

10. Chung CY, Wong DS, Li KK. Is it necessary to cover the macular hole with the inverted internal limiting membrane flap in macular hole surgery? A case report. BMC Ophthalmol. 2015;15:115.

11. Kang SW, Ahn K, Ham DI. Types of macular hole closure and their clinical implications. Br J Ophthalmol. 2003;87:1015-1019.

12. Clark A, Balducci N, Pichi F, et al. Swelling of the arcuate nerve fiber layer after internal limiting membrane peeling. Retina. 2012;32:1608-1613.

13. Hayashi H, Kuriyama S. Foveal microstructure in macular holes surgically closed by inverted internal limiting membrane flap technique. Retina. 2014;34(12):2444-2450. doi:10.1097/IAE.0000000000000252

14. Michalewska Z, Michalewski J, Dulczewska-Cichecka K, et al. Temporal inverted internal limiting membrane flap technique versus classic inverted internal limiting membrane flap technique: a comparative study. Retina. 2015;35(9):1844-1850. doi:10.1097/ IAE.0000000000000555

15. Casini G, Mura M, Figus M, et al. Inverted internal limiting membrane flap technique for macular hole surgery without extra manipulation of the flap. Retina. 2017;37(11):2138-2144. doi:10.1097/ IAE.0000000000001470

16. Shin MK, Park KH, Park SW, et al. Perfluoro-n-octane-assisted single-layered inverted internal limiting membrane flap technique for macular hole surgery. Retina. 2014;34(9):1905-1910. doi:10.1097/IAE.0000000000000339

17. Morizane Y, Shiraga F, Kimura S, et al. Autologous transplantation of the internal limiting membrane for refractory macular holes. Am J Ophthalmol. 2014;157(4):861-869.e861. doi:10.1016/j.ajo.2013.12.028

18. Imai H, Azumi A. The expansion of RPE atrophy after the inverted ILM flap technique for a chronic large macular hole. Case Rep Ophthalmol. 2014;5(1):83-86. doi:10.1159/000360693
Clinical Ophthalmology

\section{Publish your work in this journal}

Clinical Ophthalmology is an international, peer-reviewed journal covering all subspecialties within ophthalmology. Key topics include: Optometry; Visual science; Pharmacology and drug therapy in eye diseases; Basic Sciences; Primary and Secondary eye care; Patient Safety and Quality of Care Improvements. This journal is indexed on PubMed
Central and CAS, and is the official journal of The Society of Clinical Ophthalmology (SCO). The manuscript management system is completely online and includes a very quick and fair peer-review system, which is all easy to use. Visit http://www.dovepress.com/ testimonials.php to read real quotes from published authors. 